relaxation induced by TENS and interferential current in computer users with chronic nonspecific neck discomfort: An electromyographic analysis. J Back Musculoskelet Rehabil. 2015;28(1):19-24.

[2] Kroeling P, Gross A, Graham N, Burnie SJ, Szeto G, Goldsmith CH, Haines T, Forget M. Electrotherapy for neck pain. Cochrane Database Syst Rev. 2013 Aug 26;(8):CD004251.

[3] Dusunceli Y, Ozturk C, Atamaz F, Hepguler S, Durmaz B. Efficacy of neck stabilization exercises for neck pain: a randomized controlled study. J Rehabil Med. 2009 Jul;41(8):626-31.

Disclosure of Interest: None declared

DOI: 10.1136/annrheumdis-2017-eular.5843

\section{THU0710 VALIDATION OF NEW WEIGHTED SCORING SYSTEM TO PREDICT MODIFIED HEALTH ASSESSMENT QUESTIONNAIRE SCORES IN PATIENTS WITH RHEUMATOID ARTHRITIS USING NATIONAL DATABASE OF RHEUMATIC DISEASES IN JAPAN DURING 2009 AND 2014}

K. Ono ${ }^{1}$, S. Ohashi ${ }^{2}$, Y. Kadono ${ }^{3}$, H. Oka ${ }^{4}$, J. Hirose ${ }^{5}$, T. Matsumoto $^{5}$, N. Izawa ${ }^{5}$, M. Naito ${ }^{5}$, J. Nishino ${ }^{5}$, S. Tanaka ${ }^{5}$, S. Tohma ${ }^{6}{ }^{1}{ }^{1}$ Joint Surgery, Research Hospital, The institute of Medical Science, The university of Tokyo, Tokyo: ${ }^{2}$ Orthopaedic sugery, Satamihara Hospital, National Hospital Organization, Kanagawa; ${ }^{3}$ Orthopaedic sugery, Saitama medical university, Saitama; ${ }^{4} 22$ d century Medical and Research center; ${ }^{5}$ Orthopaedic sugery, Faculty of medicine, The university of Tokyo, Tokyo; ${ }^{6}$ Clinical Research center for allergy and rhuematology, Satamihara Hospital, National Hospital Organization, Kanagawa, Japan

Background: We reported the impact of joint diseases on modified health assessment questionnaire (MHAQ) scores determined using 2011 data from NinJa (National Database of Rheumatic Diseases by iR-net in Japan). The MHAQ score was significantly associated with disease in almost all joints and physical ability tended to become aggravated. We also developed a joint-weighted scoring system from odds ratios. Respective integer scores assigned to disease in bilateral and unilateral joints comprised shoulder, 4 and 2; elbow, 3 and 2; wrist, 2 and 2; hip, 0 and 3; knee, 3 and 2; ankle, 2 and 2; finger, 1 and 1. Statistical analyses indicated a cut-off at 3 points using this system (Ono K, et al., Modern Rheumatology, 2016).

Objectives: To validate the weighted scoring system using NinJa data from 2009 and 2014

Methods: We analyzed data from 7,189 and 13,459 patients listed in the NinJa database during 2009 and 2014. The presence or absence of disease in each joint (swelling and/or tenderness were considered to indicate active disease) and whether the disease was bilateral or unilateral were investigated. We calculated joint scores for each patient using a weighted scoring system and then created ROC curves for each patient based on total scores.

Results: Patients in the 2009 and 2014 groups were aged $62.4+12.6$ and $64.1 \pm 12.8$ years, median DAS28 CRP values were 2.9 and 2.4 , and total joint scores were 3.4 and 2.4 with a significant difference $(p<0.05$; Wilcoxon signedrank test) between the two groups. Analyses of ROC curves generated from scores derived using the new system and MHAQ revealed a cut-off of 3 points (same as 2011); AUC, 0.72, 0.68 (Table 1).

\begin{tabular}{lccc}
\hline Variable & $2009(\mathrm{n}=7,189)$ & $2014(\mathrm{n}=13,459)$ & $\mathrm{P}$ value \\
\hline Age (mean \pm SE, years) & $62.4 \pm 12.6$ & $64.1 \pm 12.8$ & $<0.05$ \\
Duration of disease (mean \pm SE, years) & $12.7 \pm 10.7$ & $13.0 \pm 12.9$ & 0.68 \\
MHAQ score (median) & 0.25 & 0.13 & $<0.05$ \\
DAS28 CRP (median) & 2.9 & 2.4 & $<0.05$ \\
DAS28 ESR (median) & 3.6 & 3.0 & $<0.05$ \\
Total joint score (median) & 3.4 & 2.4 & $<0.05$ \\
Joint score: ROC analysis' cut-off value & 3.0 & 3.0 & \\
AUC & 0.72 & 0.68 & \\
\hline
\end{tabular}

Conclusions: Disease activity changed from moderate to low in the NinJa cohorts and the scoring system was validated for the years 2009 and 2014. The weighted scoring system appears useful to predict functional disability in a simpler way by examining each joint rather than changes in disease activity among patients with RA.

\section{References:}

[1] Ono K, et al., The impact of joint disease on the Modified Health Assessment Questionnaire scores in rheumatoid arthritis patients: A cross-sectional study the National Database of Rheumatic Diseases by iR-Net in Japan. Modern Rheumatology, 2016(4): 529-533.

Disclosure of Interest: None declared

DOI: 10.1136/annrheumdis-2017-eular.3605

\section{THU0711 IMPACT OF SPORTS PRACTICE IN PATIENTS WITH ANKYLOSING SPONDYLITIS: A SYSTEMATIC REVIEW OF RANDOMIZED CONTROLLED STUDIES}

L. Bouleau $^{1,2,3}$, C. Palazzo ${ }^{4,5}$, M. Kerzoncuf ${ }^{1,3}$, T. Pham ${ }^{2,3} .{ }^{1}$ Physical Medicine and Rehabilitation Department, la Timone Hospital, AP-HM, ${ }^{2}$ Rheumatology
Department, Sainte-Marguerite Hospital, AP-HM; ${ }^{3}$ Aix-Marseille University, Marseille; ${ }^{4}$ Service de rééducation et de réadaptation de l'appareil locomoteur et des pathologies du rachis, Cochin Hospital, AP-HP; ${ }^{5}$ ECaMO, INSERM U1153, Paris, France

Background: The recent update of the ASAS-EULAR management recommendations for axial spondyloarthritis highlights effectiveness of home exercises and physical therapy. However, the adherence rates to these structured exercises are low, what could limit their long-term effectiveness. An option could be to replace or to complete the practice of specific exercises with the regular practice of a recreational sport activity. Data concerning the effectiveness and tolerance of sports in ankylosing spondylitis (AS) are scarce.

Objectives: To assess the tolerance and effectiveness of recreational sports practice on function, spinal mobility, disease activity, pain and quality of life in patients with AS.

Methods: A systematic review was performed according the PRISMA guidelines, using the following search engines: MEDLINE (Pubmed), COCHRANE Library, Google Scholar and Physiotherapy Evidence Database (PEDro), completed by a manual research. We included randomized controlled trials (RCTs) from 1984 to September 2016, with participants with AS fulfilling the New York modified criteria, and interventions involving sports practice. We defined "sports" according to the $\mathrm{MeSH}$ definition as "Activities or games, usually involving physical effort or skill [with purpose of] pleasure, competition, and/or financial reward." Exercise therapy and physiotherapy were not included in the research. The outcomes measured were the followings: stiffness/spinal mobility, function, disease activity, quality of life, pain, side effects. Methodological quality was assessed using the PEDro scale.

Results: From the 40 studies identified after screening, only 5 articles were selected for eligibility. One study was excluded (poor quality). The 4 RCTs concerned a total of 187 AS patients. Interventions were Pilates, Tai Chi, swimming, walking, and exergames. Control groups received either no specific intervention or standard exercise therapy. The studies were too heterogeneous to be pooled but the comparisons inter and intra-groups in each study tended to show significant effectiveness of sports practice on function, activity and spinal mobility. Data on tolerance were scarce, but no significant side effect was reported.

Conclusions: Recreational sports practice may improve function, activity and spinal mobility in patients with AS. However, there are very few studies available, and evidence-level is low. More controlled randomized clinical trials should be conducted in the future to confirm the effectiveness and safety of sports in AS.

Disclosure of Interest: None declared

DOI: 10.1136/annrheumdis-2017-eular.4968

\section{THU0712 DUTCH NORM SCORES FOR FOUR DUTCH-FLEMISH PROMIS ITEM BANKS IMPORTANT FOR PATIENTS WITH RHEUMATOID ARTHRITIS}

M.H. Crins ${ }^{1}$, L.D. Roorda ${ }^{1}$, H.C. de Vet ${ }^{2}$, R. Westhovens ${ }^{3,4}{ }^{\text {, M. Boers }}{ }^{2,5}$, J. Dekker ${ }^{6}$, C.B. Terwee ${ }^{2}$. ${ }^{1}$ Amsterdam Rehabilitation Research Center, Reade; ${ }^{2}$ Department of Epidemiology and Biostatistics, The EMGO Institute for Health and Care Research, VU University Medical Center, Amsterdam, Netherlands; ${ }^{3}$ Rheumatology, University Hospitals; ${ }^{4}$ Department of Development and Regeneration, Skeletal Biology and Engineering Research Center, KU Leuven, Leuven, Belgium; ${ }^{5}$ Amsterdam Rheumatology and immunology center; ${ }^{6}$ Department of Rehabilitation Medicine and Department of Psychiatry, VU University Medical Center, Amsterdam, Netherlands

Background: In the assessment of patients with rheumatoid arthritis (RA) it is important to measure physical function, fatigue, pain behavior and pain interference. The Patient-Reported Outcomes Measurement Information System (PROMIS) initiative developed item banks for measuring these concepts. These item banks were translated into Dutch-Flemish language.

Objectives: To facilitate interpretation and implementation of PROMIS in the Netherlands, we aimed to calibrate and validate the Dutch-Flemish PROMIS Physical Function (DF-PROMIS-PF), Fatigue (DF-PROMIS-FA), Pain Behavior (DF-PROMISPB) and Pain Interference (DF-PROMIS-PI) Item Banks in the general Dutch population, to obtain Dutch norm scores for these item banks.

Methods: 3365 persons of the general Dutch population completed a web-based survey, of which 1309 persons completed the full DF-PROMIS-PF (121 items), 1007 persons completed the full DF-PROMIS-FA (95 items), and 1049 persons completed the full DF-PROMIS-PB (39 items) and the full DF-PROMIS-PI (40 items). The sample was stratified for gender, age, education, and ethnicity according to the distribution of the 2015 census of the general Dutch population. A one-factor confirmatory factor analysis (CFA) was performed per item bank to assess unidimensionality. A graded item response model (GRM) was fitted per item bank to evaluate the item characteristics of the item banks and to facilitate future development of computer adaptive tests (CATs). Ordinal regression models were used to evaluate Differential Item Functioning (DIF) for language (Dutch vs. English) as a measure of cross-cultural validity.

Results: All four item banks showed good fit to the GRM: they showed good fit indices for CFA and high percentages of explained variance by first factor. The item banks showed only little local dependency and the scalability coefficients suggested strong scalability for all four item banks. The item characteristics 